\title{
Sur les confluences souterraines
}

\author{
Par Marcian Bleahu ${ }^{1}$ )
}

Avec 16 figures dans le texte

Un réseau souterrain comprend la totalité des vides qui se trouvent en connexion, et dont la formation est due à l'action de l'eau. Outre les espaces vides accessibles à l'homme, il comprend également toutes les voies par lesquelles l'eau circule et qui sont inaccessibles à cause de leurs dimensions réduites. En partant de la prémisse que les espaces souterrains vides sont créés par la circulation de l'eau, on pourrait comparer un réseau souterrain à un réseau hydrographique de surface. En réalité, la comparaison ne saurait aller trop loin car la manière dont se forment ces deux types de réseau est tout à fait différente. Afin de mettre en évidence cette différence, il faut commencer par analyser la manière dont se constituent les réseaux hydrographiçues à la surface de la terre.

\section{Les réseaux hydrographiques de surface}

Les eaux de surface ont la tendance bien connue de confluer,formant ainsi des réseaux hydrographiques unitaires. La naissance des eaux affluentes d'un cours d'eau est due aux inégalités de terrain, qui déterminent la concurrence des lignes de la plus grande pente. Lorsque les eaux de précipitation s'écoulent sur une pente, elles y forment de petites rigoles dans lesquelles s'accumule graduellement l'eau de ruissellement. Les rigoles sont constamment élargies et creusées par l'érosion et au fur et à mesure de leur élargissement elles collectent des quantités croissantes d'eaux. De cette manière se forme un lit fixe qui constitue le début d'un torrent ou d'un ruisseau. En même temps, l'eau qui s'écoule sur les versants vers le lit fixe creuse d'autres rigoles qui, dans leur évolution donnent naissance à d'autres lits fixes, donc des torrents et des ruisseaux affluents du premier. Ils forment ensemble un réseau hydrographique. Le premier facteur qui détermine la constitution d'un réseau hydrographique est donc l'écoulement diffus de l'eau sur les versants.

1) Institut géologique de la Roumanie, Bucarest. 
C'est l'érosion régressive qui représente le second facteur; le mécanisme en est le suivant (fig. 1) $)^{2}$ ): le long d'un cours d'eau $\mathrm{AB}$, à l'endroit C, l'érosion verticale creuse le lit jusqu'à C'. Grâce à ce surcreusement la pente diminue vers l'aval et augmente vers l'amont. Par conséquent, se produit un déséquilibre à l'endroit situé immédiatement en amont de $\mathrm{C}^{\prime}$, où le point $\mathrm{D}$ aura la tendance à atteindre le niveau de $\mathrm{C}^{\prime}$; ce fait aura pour conséquence le déplacement du déséquilibre encore plus en amont. Le déplacement progressif vers l'amont du point de déséquilibre entraine un déplacement de l'érosion, d'où le nom d'érosion régressive, ou remontante (Macar, 1946).

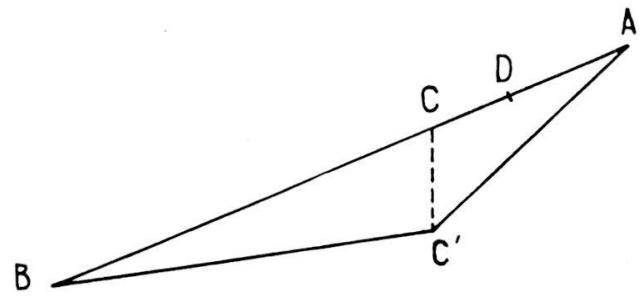

Fig. 1. Mécanisme de l'érosion verticale et régressive.

L'érosion régressive contribue à la modification des versants en déséquilibrant les particules solides des pentes, ce qui favorise la formation des rigoles et, par conséquence, l'extension latérale d'un réseau hydrographique, ainsi que le déplacement en recul des sources d'un cours d'eau à lit fixe.

Dans un cas plus complexe, l'érosion successive en amont des points du thalweg de la rivière C (fig. 2) entraine le surcreusement jusqu'à la première source de celle-ci (b); ensuite commence l'action de l'eau ruissellement qui, érodant progressivement vers l'amont, prolonge le thalweg. Dans le cas particulier de la fig. 2, le déplacement des sources de la rivière $\mathrm{C}$ peut avoir lieu jusqu'au trajet de la rivière $\mathrm{B}$, qui sera captée. Le réseau hydrographique de la rivière $\mathrm{A}$ comprendra dorénavant tout le bassin de la rivière $\mathrm{C}$, en amont du point de confluence $\mathrm{C}^{\prime}$.

Les considérations ci-dessus permettent la déduction de quelques traits caractéristiques des réseaux hydrographiques de surface:

1. Un réseau hydrographique comprend la totalité des cours d'eau à drainage commun. Dans les limites d'un réseau, le cours d'eau se trouvant au niveau le plus bas, est le plus important et joine le rôle

$\left.{ }^{2}\right)$ Les figures ont été exécutées par M. G. Nicolesco, auquel j’adresse, par cette voie, mes vifs remerciements. 
d'un collecteur général, tandis que le rang des affluents diminue avec leurs dimensions (affluents des affluents).

2. Un réseau hydrographique prend naissance de l'écoulement diffus des eaux sur les versants, ce qui a pour conséquence la f'ormation des lits fixes évoluant en rivières. Sur les versants de ces dernières,

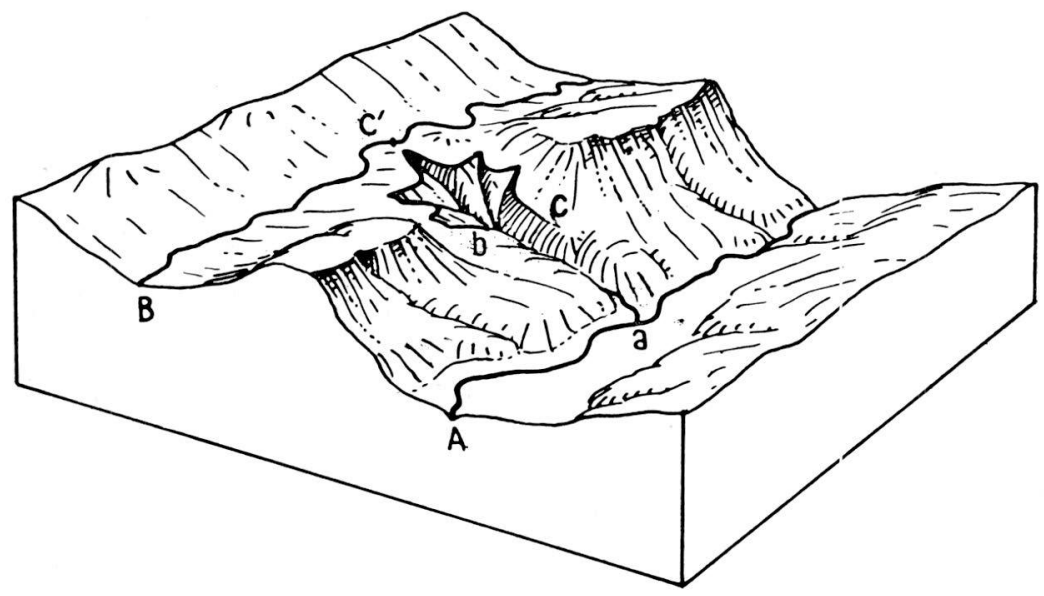

Fig. 2. Schéma d'une capture de surface.

l'écoulement diffus creuse d'autres lits fixes, donc d'autres rivières, affluentes des premières, et ainsi de suite.

3. Un rôle important revient à l'érosion régressive, qui contribue à la formation des lits fixes sur les versants et, par l'action linéaire longitudinale, produit un allongement des rivières, par recul des sources.

4. Un réseau hydrographique a une tendance constanta d'extension, en occupant une superficie aussi grande que possible. Ceci conduit à une concurrence entre les réseaux avoisinants, dont sort vainqueur le réseau au niveau de base le plus bas.

\section{Considérations générales sur les réseaux souterrains}

Examinons d'abord la mesure dans laquelle les élénents établis pour les réseaux de surface, peuvent être appliqués aux réseaux souterrains.

La première loi, concernant la relation entre la grandeur d'un cours d'eau et le niveau altimétrique est en général également valable en souterrain, mais elle n'est guère universelle. On peut rencontrer, par 
exemple, des affluents secondaires à débit supérieur et ayant un lit donc une galerie - plus grand que celui du collecteur. Une telle situation, impossible dans les limites de l'érosion fluviatile de surface, (mais bien possible, par exemple, dans le cas d'une confluence entre une vallée fluviatile et une vallée glaciaire) prouve que la confluence des deux cours d'eau souterrains s'est produite par hasard et qu'elle est de date récente. Ce premier exemple nous avertit qu'en souterrain il existe d'autres lois qui régissent la constitution de réseaux hydrographiques.

Ce fait est mieux mis en évidence si l'on essaye d'appliquer en souterrain la seconde loi. En effet, l'écoulement diffus sur le versants, facteur essentiel dans l'établissement de réseaux de surface, n'est point concevable en souterrain. Là il n'existe guère d'écoulements sur les versants, qui puissent créer des rigoles et des lits fixes, évoluant en affluents, car il n'existe pas d'eau de ruissellement à écoulement en nappe, comme il n'existe non plus des précipitations ni des roches désagrégées qui permettent l'établissement d'un lit sur un trajet fixe. L'eau circule là par des canaux préexistants, imposés par le litage et la tectonisation des roches. L'eau qui suinte sur les parois des galeries n'est en rien comparable à l'écoulement diffus des versants de surface et elle ne joue aucun rôle dans la formation des réseaux souterrains.

L'érosion régressive présente, elle aussi, des particularités en souterrain. Supposons que A. B, C de la fig. 2 sont des rivières souterrains, donc évidemment à cours fixés par des galeries. La rivière $\mathrm{C}$ subira une érosion régressive, déterminée par le niveau de base local représenté par la confluence de $a$. L'érosion régressive se propagera en amont jusqu'à la source de C, c'est-à-dire jusqu'à l'endroit où la rivière souterraine prend ses eaux dans un cours d'eau de la surface.

Vers son origine, un cours d'eau souterrain peut se diviser en plusieurs cours plus petits, qui eux aussi coulent par des canaux fixes aboutissant à la surface. Une telle ramification n'est guère comparable à une source de surface, où en fin de compte il existe une zone à écoulement diffus, sans lits fixes et où l'eau de ruissellement crée de nouveaux lits, déterminant un recul du lit et l'allongement de la rivière. Autrement dit, pour le ruisseau souterrain C la source $b$ doit être à la surface et elle ne saurait capter la rivière souterraine B.

Il ne peut donc exister en souterrain un recul de la source, l'érosion régressive n'étant pas en état d'ouvrir de nouvelles voies de circulation. Elle ne crée pas de nouvelles confluences, mais exerce son activité seulement sur les trajets existants, tendant à établir le profil d'équilibre d'un cours d'eau existant. 
Enfin, la quatrième loi ne peut ètre appliquée, elle non plus, aux réseaux souterrains. Ceux-ci, étant constitués par des lits fixes, n'ont guère la tendance d'extension latérale au détriment d'autres réseaux avoisinants. L'extension est toutefois possible, par captures latérales, mais celles-ci sont plutôt un effet dû au hasard et non pas une nécessité résultant d'un mécanisme qui s'imposerait comme une loi.

Les observations ci-dessus mènent à la conclusion que l'organisation d'un réseau hydrographique souterrain doit être déterrninée par des processus complètement différents par rapport à ceux de la surface, des processus basés sur des facteurs différents.

La constitution d'un réseau hydrographique souterrain complexe dépend de la mesure dans laquelle peuvent se réaliser des confluences entre différents cours d'eau. Du moment que les cours d'eau ont des voies préétablies, chaque cours d'eau devrait couler théoriquement par un canal indépendant, reliant le point de pénétration de l'eau en souterrain, au point de l'exurgence. Néanmoins, les confluences souterraines existent et nous avons vu que les processus morphosculpturaux et hydrodynamiques ne peuvent guère réaliser des confluences de la manière dont ce phénomène se produit en surface. Pour commencer, nous pouvons donc conclure que la formation des corfluences souterraines n'est pas conditionnée par des processus propres, déterminés par l'écoulement mème de l'eau, comme en surface, mais qu'elle a un caractère fortuit et dépend du concours de certaines circonstances indépendantes du phénomène de l'écoulement de l'eau.

Les réseaux des cours d'eau souterrains présentent deux traits caractéristiques, qui les distinguent des réseaux superficiels:

1. L'eau coule seulement par des canaux fixes, qu'elle ne crée point, mais qui sont imposés par le litage et par la tectonisation des roches.

2. Les réseaux souterrains se développent dans un espace tridimensionnel, ce qui rend possible la coexistence de plusieurs cours d'eau superposés appartenant à un seul réseau ou à des réseaux indépendants.

Ces deux caractéristiques indiquent les facteurs qui conditionnent les confluences souterraines: préexistence des voies initiales s'intersectant et mode de distribution de ces voies dans l'espace. C'est en fonction de ces deux éléments que nous essayerons de faire une classification des confluences souterraines. 


\section{Classification des confluences souterraines}

\section{Confluences dues à l'intersection des voies initiales}

1. Toute discontinuité dans la masse du calcaire représente des voies favorables à la circulation de l'eau. Les premières à envisager à ce point de vue sont les fissures.

Un massif calcaire, lithologiquement homogène, présente un système de fissures distribuées de manière uniforme, représentant autant de canaux capillaires, donc des voies d'accès de l'eau dans la massif. Supposons un tel système de fissures dans lequel l'eau pénètre par les points 1-7 (fig.3). S'il n'existe pas un point d'écoulement, le système en entier demeure sous pression, l'eau envahissant tous les capillaires, où elle reste stagnante. Si l'un des canaux débouche à la surface dans le point 8 (par ex. par le démantelement du calcaire), l'eau est mise en mouvement et commence à couler. Si la sortie de l'eau se fait difficilement, le système tout entier demeure sous charge, l'écoulement se fait lentement et la corrosion travaille dans tous les canaux en les élargissant graduellement. Il n'existe guère de rivière souterraine dans le réseau, le déplacement de l'eau ayant lieu dans l'ensemble du réseau, comme une masse unitaire.

Si les canaux ont été élargis, nous aurons une caverne-labyrinthe, avec un système compliqué de galeries, à intersections nombreuses, à confluences multiples et dépourvu d'un collecteur principal. Nous désignerons ce type sous le nom de confluences en réseau-labyrinthe.

Si dans le réseau de la fig.3 le drainage de l'eau se produit aisément, l'eau qui pénètre par les points 1-7 cherche les voies les plus courtes, présentant les pentes les plus inclinées. En utilisant ces voies, elle les élargit, le système passe de l'écoulement sous charge à un écoulement à niveau libre et l'eau quitte les petits canaux ascendants ou en cul-de-

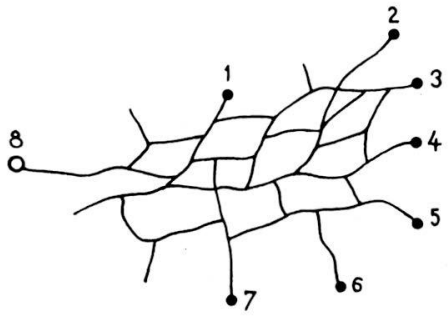

Fig. 3

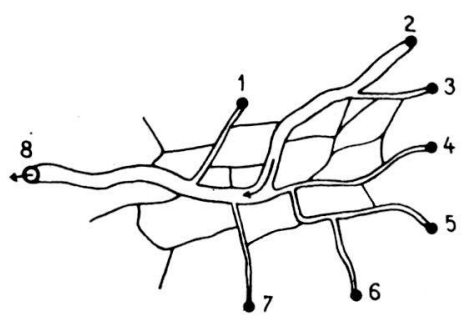

Fig. 4

Fig. 3. Confluences dans un réseau-labyrinthe. Fig. 4. Confluences dans un réseau dentritique. 
sac (fig. 4). Si l'un des points d'absorption, par ex. le point 2, reçoit un débit plus grand, l'évolution de la galerie 2-8 sera plus rapide et elle deviendra une sorte de collecteur principal par rapport aux autres cours d'eau qui prendront l'aspect d'affluents. Ceci ne représente qu'une image morphologique, car au point de vue fonctionnel, tous les cours d'eau sont égaux, ce qui n'est pas le cas dans les ríseaux de surface, où la rivière principale détermine la formation des affluents. Nous désignons ce type sous le nom de confluences en résecau dendritique.

2. Un autre type des discontinuités dans la roche est représenté par les dislocations avec déplacement des flancs: diaclases, failles, décrochements. Ceux-ci constituent des voies ouvertes dès le début dans le massif calcaire, que les cours d'eau adopteront comme telles. Dans ce cas la phase d'écoulement sous charge peut manquer, les cours d'eau adoptant les voies les plus courtes entre le point d'adsorption et celui d'exurgence. Étant donné que les efforts tectoniques créent des systèmes de dislocation à orientation déterminée dans l'espace, les galeries de grottes développées à leur dépens ont des orientations régulières, à confluences en réseau orthogonal ou rhomboïdal (fig. 5).

3. Le troisième type de discontinuités dans la roche est représenté par les joints de stratification.

Une simple discontinuité déterminée par la stratification n'est pas nécessairement favorable à la formation des confluences, car l'eau qui

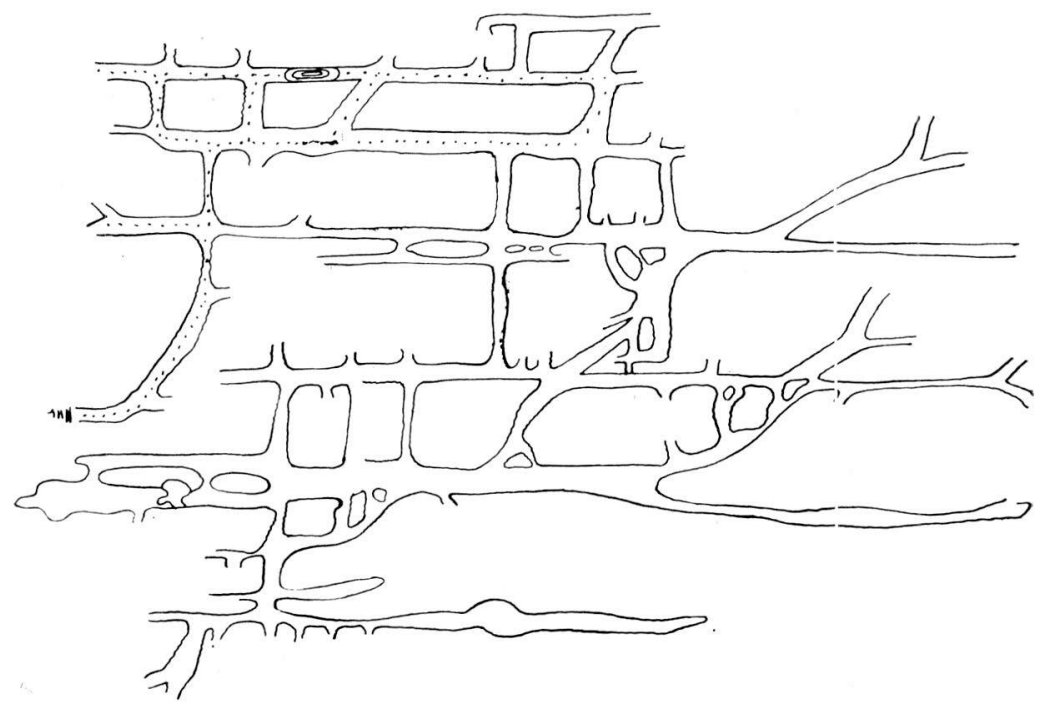

Fig. 5. Confluences dans un réseau orthogonal. 


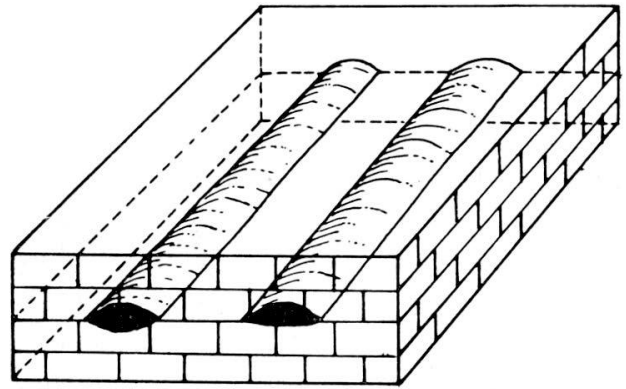

Fig. 6. Galeries indépendantes sur la surface d'une couche.

pénètre à la surface d'une couche circule le long de celle-ci, élargissant son canal; son trajet est parallèle à celui d'autres cours d'eau utilisant la même surface, mais à trajet indépendant (fig. 6). Cependant, lorsque les couches sont plissées, les cours d'eau étant dirigés par le pendange, il a y plus de chances qu'ils se rencontrent (fig. 7). Le plissement ne représente qu'un cas particulier. En général, tout élément qui détermine la convergence de quelques voies potentielles d'écoulement d'eau (par ex. fissures ou failles), sur la surface d'une couche, mène à des confluences sur cette surface.

Le cas limite des confluences contrôlées par la stratification est celui du développement des réseaux hydrographiques au contact $\mathrm{du}$ massif calcaire et de la couche soujacente imperméable. Au cas d'un massif calcaire exhaussé, dont la base se trouve au-dessus du niveau hydrostatique général, les eaux traversent verticalement le massif en

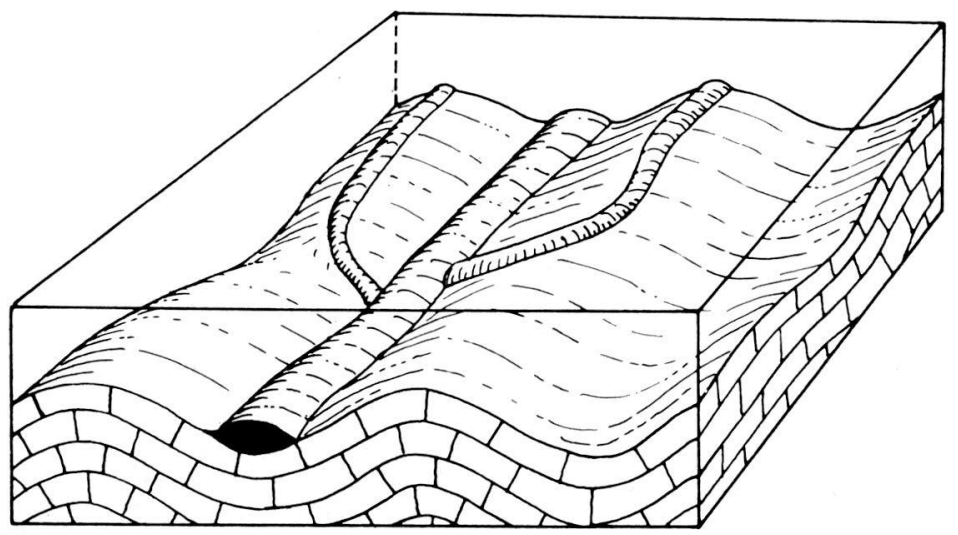

Fig. 7. Confluences déterminées par le plissement des couches. 
entier et coulent ensuite sur la surface de la couche imperméable, jusqu'à ce qu'elles surgissent à la surface. Les eaux peuvent traverser le calcaire par des canaux indépendants, sans confluer, mais une fois arrivées à la surface imperméable, la probabilité de se rencontrer est assez grande, cette surface jouant le rôle d'un collecteur généraldes eaux. La rencontre des eaux à la surface de la roche imperméable générera des confluences de contact stratigraphique. De telles confluences ne sont guère limitées à la surface de contact entre le calcaire et son soubassement imperméable; elles peuvent prendre naissance sur n'importe quelle couche imperméable intercalée aux calcaires, par ex. un banc, même très mince, mais continu, d'argiles.

\section{Confluences dues à la position dans l'espace des voies de circulation}

Le fait que les canaux qui dirigent la circulation de l'eau dans le karst sont couverts, crée la possibilité que ceux-ci soiert non seulement situés à un même niveau, mais qu'ils soient aussi superposés et indépendants. Les processus d'évolution normale qui produisent l'élargissement des espaces vides, peuvent toutefois déterminer la rencontre de ces canaux juxtaposés et superposés, donc la naissance des confluences.

1. L'un des processus qui peuvent engendrer de telles confluences est le surcreusement. Il en résulte des confluences de surcreusement. Par le canal supérieur de la fig. 8 a coule une riviére $\mathrm{A}-\mathrm{B}$; par le canal inférieur - une rivière plus petite $\mathrm{C}-\mathrm{D}$. La rivière $\mathrm{A}-\mathrm{B}$, plus active, a une puissance d'érosion supérieure et creuse fortement le lit, jusqu'à ce qu'elle intersecte la galerie $\mathrm{C}-\mathrm{D}$. Lorsque le lit de la rivière $\mathrm{A}-\mathrm{B}$ arrive au niveau de la branche $\mathrm{C}$ de la galerie inférieure, l'eau de cette dernière

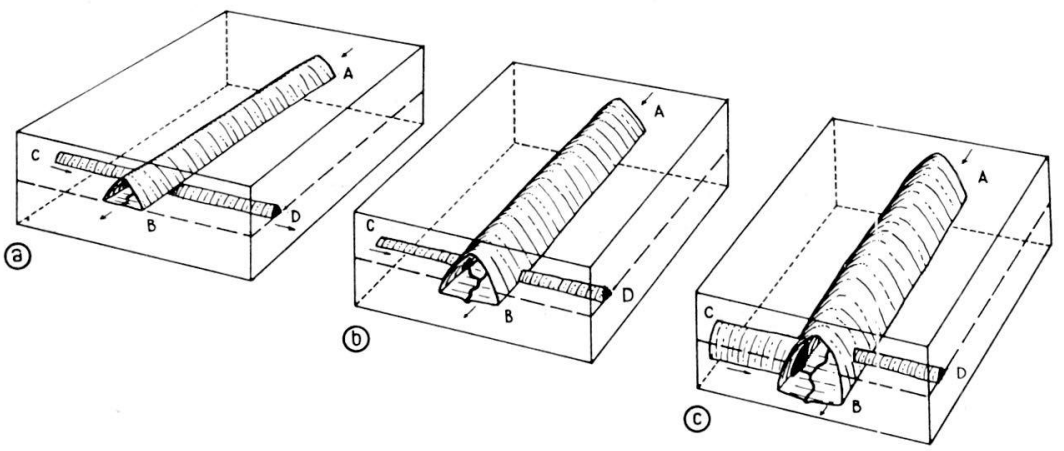

Fig. 8. Confluences par surcreusement dû à l'intersection de deux galeries. 
s'écoule dans la rivière $\mathrm{A}-\mathrm{B}$ et celle-ci, à son tour, toujours par B (fig. 8 b), tandis que par la branche D il ne coule plus d'eau. L'érosion progressant, ce sont seulement les galeries A-B et la branche $\mathrm{C}$ qui continuent à être surcreusées, la branche $\mathrm{D}$ restant suspendue, car il n'y coule plus d'eau pour la creuser d'avantage (fig. 8c). De cette manière s'explique beaucoup de galeries "suspendues», dont l'ouverture se trouve quelque part dans la partie supérieure de la paroi d'une galerie et dont l'accès est souvent difficile. D'habitude, ces galeries suspendues doivent avoir en vis-à-vis, de l'autre côté de la galerie principale, une continuation, mais ceci n'est point obligatoire, car, ainsi qu'il résulte de la fig. 9, les deux galeries au début indépendantes et superposées $(a)$, peuvent avoir, sur une portion, un trajet parallèle et dans le même plan vertical. Après le surcreusement de la galerie principale supérieure (phase $b$ ), les deux segments C et D débouchent dans la galerie $\mathrm{AB}$ à quelque distance, de sorte qu'apparemment la galerie suspendue D n'aura guère de continuité en vis-à-vis de l'emmergence de la galerie D.

L'intersection par surcreusement peut mener également à des confluences multiples, comme dans la fig. 10. C'est l'un des cas où l'on a l'impression qu'il s'agit du recoupement d'un méandre.

Tous les cas mentionnés ont pour caractère commun l'intersection de quelques voies préexistantes indépendantes; de ce fait nous parlerons donc de confluences antécédentes, toutes les voies étant préexistantes.

Le surcreusement d'une galerie peut entraîner aussi d'une autre manière la formation des confluences souterraines. La fig. 11 montre un niveau supérieur avec une galerie dont la rivière souterraine a la tendance d'approfondir son lit par érosion. A un niveau inférieur se trouve un réseau de petits canaux sous charge, où l'eau pénétrant par les points $\mathrm{C}$ et $\mathrm{D}$ est stagnante à défaut d'écoulement. Par surcreusement, la galerie A-B arrive à intersecter le réseau sous charge. En lui procurant des possibilités d'écoulement, l'eau de ce réseau se met en mouvement. Lorsque l'alimentation par $\mathrm{C}$ et D est continue, les segments $\mathrm{CC}^{\prime}$ et $\mathrm{DD}^{\prime}$ deviennent des galeries actives, d'abord sous charge et ensuite à niveau libre. Ayant maintenant un écoulement direct, l'eau ne comble plus le reste du réseau, qui n'évolue plus. A la longue, un réseau de rivières souterraines prendra donc naissance avec une rivière principale et deux affluents.

Vu que la galerie supérieure surcreusée n'a pas rencontré au-dessous de galeries formées, mais seulement une structure favorable au développement de canaux, nous parlerons,le cas écheant, de confluences épigénétiques. 


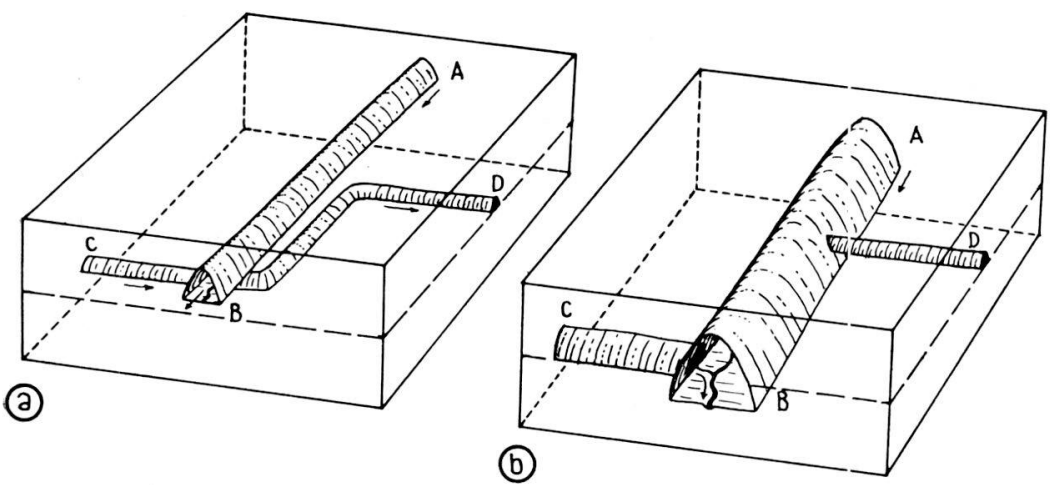

Fig. 9. Confluences par surcreusement avec superposition partielle de deux galeries.

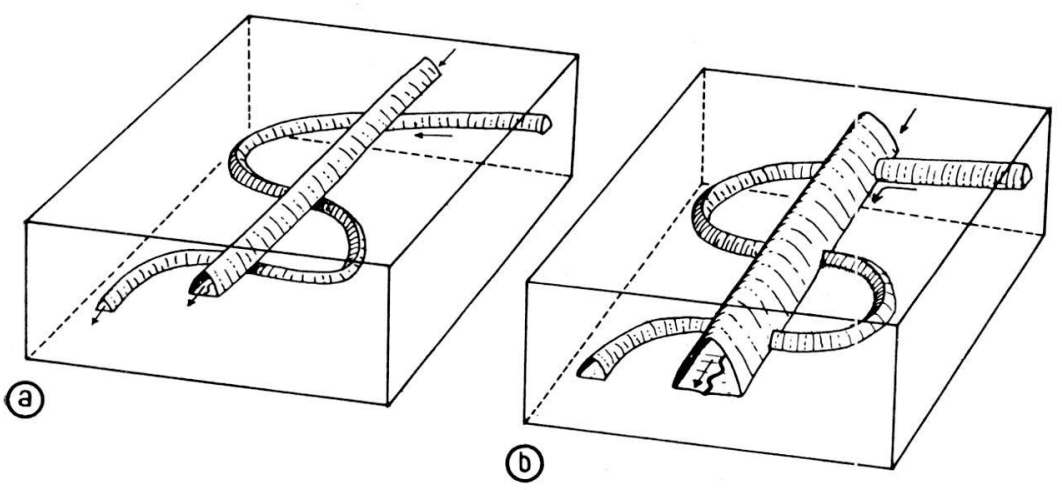

Fig. 10. Confluences multiples par surcreusement.

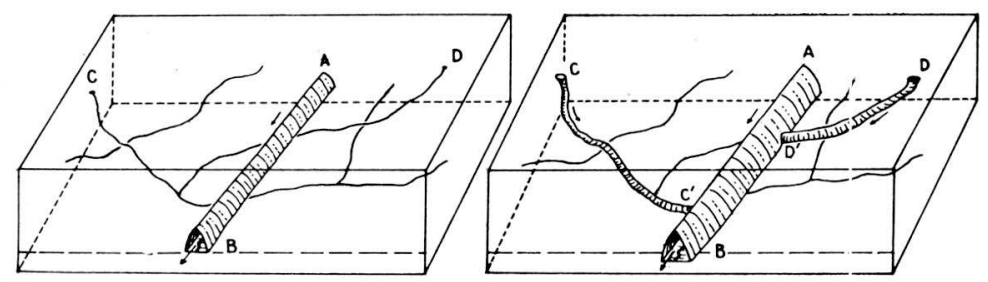

(a)

(b)

Fig. 11. Confluences épigénétiques dues à l'activation par surcreusement, des fissures capillaires. 


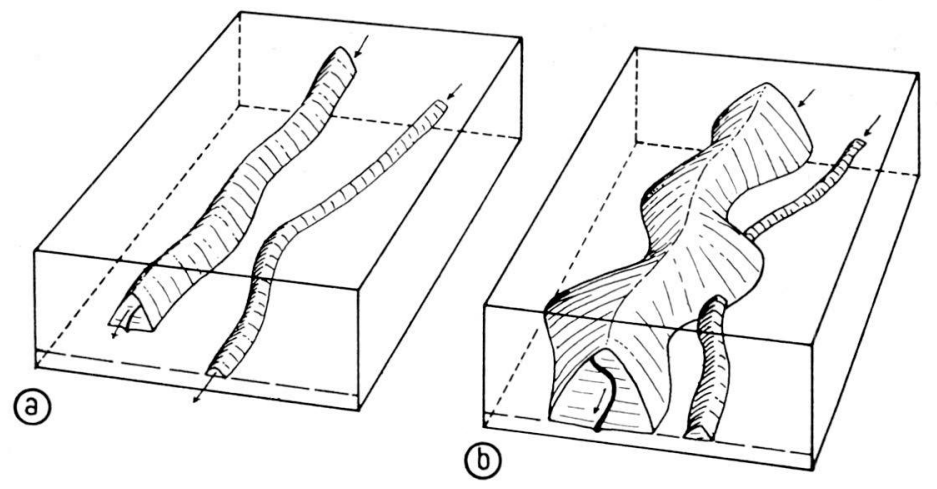

Fig. 12. Confluences antécédantes par érosion latérale.

2. Le surcreusement n'est qu'un aspect particulier du processus général d'élargissement des canaux souterrains, étant dû exclusivement à l'érosion verticale exercée par le cours d'eau. Cependant l'eau exerce son activité également par érosion latérale, surtout dans les boucles des méandres. Grâce à cette «conquête» latérale de l'espace, un cours d'eau peut rencontrer un autre canal, qu'il doit intersecter, produisant une confluence toujours du type antécédent (fig. 12); mais il peut aussi intersecter un système de fissures inactives qu'il activera par la possibilité qu'il crée à l'eau de s'écouler, générant ainsi des confluences du type épigénétique (fig. 13).

3. Un troisième mode d'élargissement des espaces vides est de bas en haut. Ce processus peut avoir lieu par corrosion, pendant la phase de conduite forcèe, mais aussi par érosion («érosion remontante» de

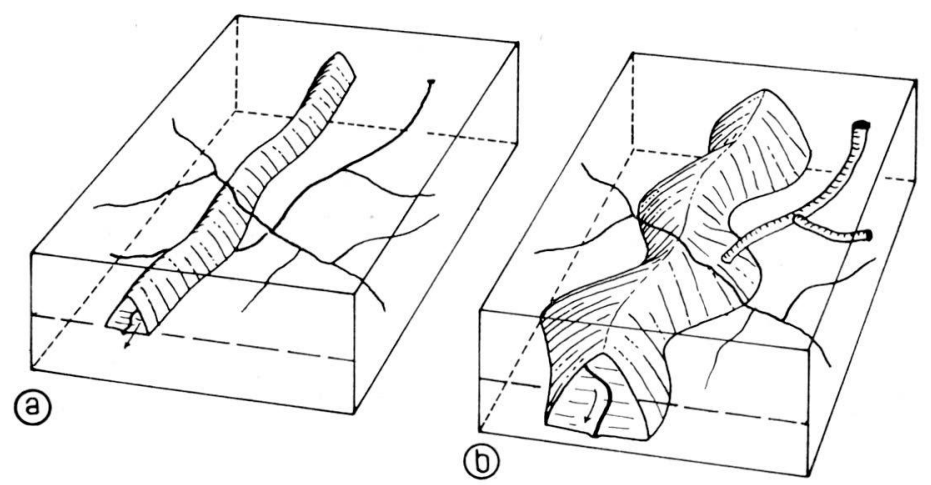

Fig. 13. Confluences épigénétiques par creusement latéral. 


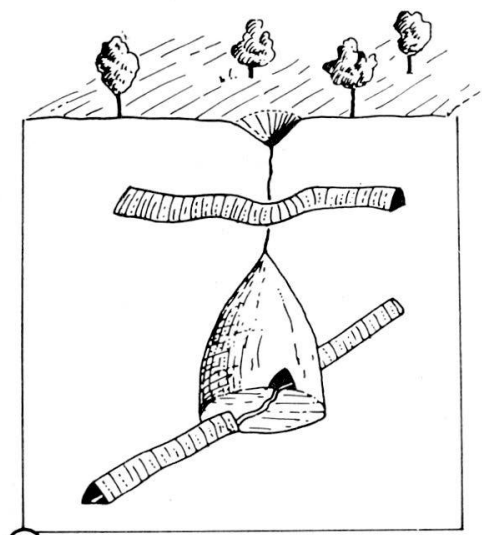

(3)

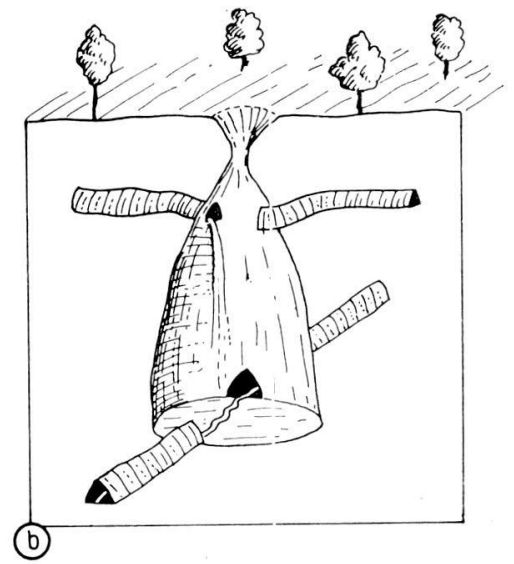

Fig. 14. Confluence antécédante par érosion remontante.

B. Gèze). Dans ce cas aussi la galerie inférieure peut rencontrer une galerie supérieure préexistante, formant une confluence antécédente (fig. 14) ou bien seulement des fissures inactives qui seront activées, évoluant en affluents et formant une confluence épigénétique (fig. 15).

L'élargissement de l'espace vide vers le haut est dû le plus souvent aux affaissements. Ces derniers peuvent être dûs au recul des parois (par l'érosion latérale ou effondrement), ce qui mène à l'elargissement de l'arc de voûte jusqu'à son instabilité, ou bien à l'attaque directe de la voûte par l'altération endochtone ou l'élargissement des fissures

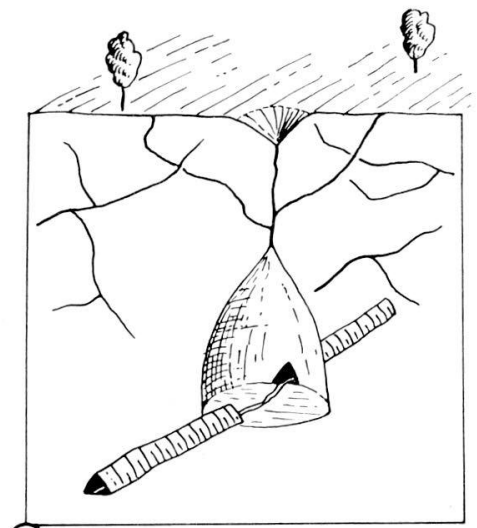

(2)

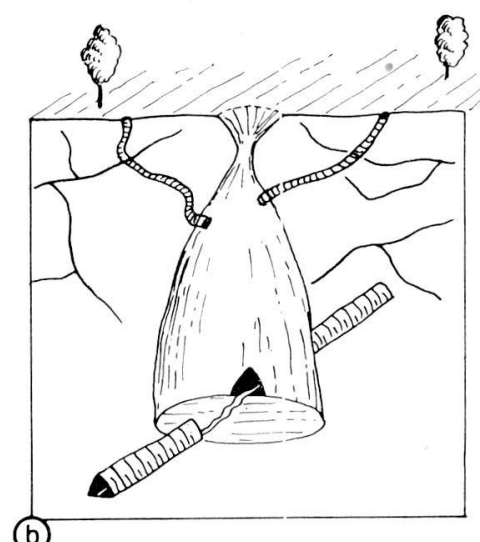

(b)

Fig. 15. Confluences épigénétiques par érosion remontante. 
causé par l'eau d'infiltration. L'effondrement peut réaliser la jonction avec un système de galeries supérieures indépendantes, ce qui donne naissance à des confluences par effondrement. C'est le cas des galeries qui débouchent dans les parois d'une salle haute, ayant une continuation dans la paroi en vis-à-vis (fig. 16). Le phénomène peut se produire dans des galeries actives lorsque la rivière supérieure devient un affluent de la rivière inférieure, mais aussi dans les réseaux fossiles, lorsque "la confluence» est seulement une rencontre des galeries dépourvues d'eau et non pas une confluence hydrologique.

Les effondrements peuvent mener également au débouchement de quelques capillaires inactifs, susceptibles d'être réactivés. «L'invasion» d'un affluent supérieur, mentionnée par Ph. Renault (1958, fig. 7/4, en bas), doit être considérée également comme la conséquence d'un tel phénomène. A défaut du stade de fissure réactivée, "l'invasion», telle qu'elle est présentée, n'est guère concevable, car on doit se demander : où donc s'écoulait l'eau du cours supérieur avant la réalisation de la confluence?

Quant au «soutirage» mentionné toujours par Renault (1958, fig. 7/4, en haut), il est simplement impossible dans l'écoulement libre, car qui donc aurait créé la galerie inférieure? Elle doit avoir préexisté, car aussi molle que soit une couche, l'eau n'y peut pas actionner comme un bélier pour se frayer une voie. Le problème du soutirage nous conduit à l'analyse du dernier type de confluence souterraine.
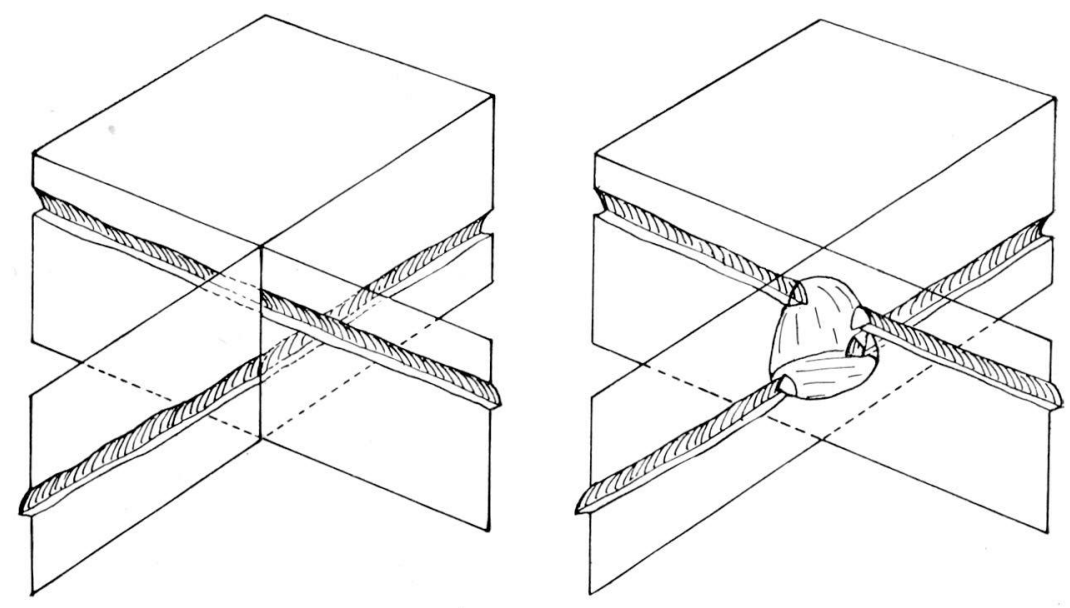

Fig. 16. Confluence par effondrement. 
III. Confluences dues aux processus hydrodynamiques

Tous les types de confluences analysées jusqu'à présen` ont un trait commun: ils ont un caractère incident et dépendent de la probabilité d'intersection des voies de circulation préexistantes, fussent-elles actives ou potentielles. Il semblerait donc que la réalisation, dans un massif karstique, d'un réseau hydrographique souterrain a quelque chose du fortuit, fait démontré d'ailleurs par la possibilité de coexistence, dans le même massif, de plusieurs réseaux indépendants (démonstration faite par ex. lors du creusement du turnel de Mont d'Or - citée par O. Lehman). Mais le plus souvent c'est le cas inverse qu'on trouve.

On prétend généralement que le karst mène à la désorganisation des réseaux hydrographiques de surface, du fait que les scutirages (les captures souterraines) provoquent le démembrement d'un réseau épigé unitaire. On constate cependant que, bien que les points d'absorption de l'eau soient multiples, les résurgences sont relativement peu nombreuses, ce qui mène implicitement à la conclus on que dans le massif calcaire a lieu une "réorganisation» du réseau hydrographique, c'est à dire une confluence des eaux qui ont pénétré indépendamment dans la calcaire. Cette réorganisation étant presque une loi, peutelle être mise seulement au compte du hasard, de la possibilité d'intersection des voies de circulation? Evidemment non, ce qui prouve qu'il existe également des processus particuliers, déterminés par l'écoulement-même de l'eau et qui mènent à la réalisation des confluences souterraines.

Analysant un réseau souterrain dendritique, l'on constate qu'il s'agit d'un canal collecteur qui, en aval, débouche à la surface par une exurgence et qui en amont se ramifie en affluents, qui er. surface ont leur origine dans des ponors. L'impression que donne un tel réseau est que le canal collecteur exerce sur les eaux pénétrant de la surface une sorte d'attraction, en les absorbant intégralement. Evidemment, les eaux qui proviennent de la surface n'ont aucun motif «d'envahir» le collecteur principal, tant qu'elles ne sont pas dirigées par des éléments structuraux. Le fait qu'elles arrivent quand même dans le collecteur indique que, loin d'être une manière d'expression «l'attraction» qu'excerce le collecteur sur les affluents trahit un processus génétique.

W. Knebel (1906) est le premier à essayer d'expliquer le phénomène, supposant qu'un cours d'eau souterrain exerce une attraction sur les cours d'eau annexes, par un processus analogue à celui qui se produit dans les pompes à vite. A n'importe quel endroit sur les parcours d'un canal, où il existe un rétrecissement la vitesse de l'eau augmente, 
créant une dépression qui exerce une succion sur l'eau des voies latérales. Selon Knebel, la première dépression est engendrée par l'exurgence d'un réseau souterrain. L'exurgence crée une dépression qui se déplacera en amont. La dépression attire l'eau des voies latérales qui, mise en mouvement, aura une action dissolvante sur le calcaire, élargissant les canaux de circulation. La dépression avancera par ces derniers de manière régressive, déterminant une nouvelle absorption et ainsi de suite, jusqu'à ce que l'une des voies d'eau arrive à la surface, attirant un cours d'eau épigé.

C'est de cette façon que Knebel explique la génèse des ponors et des réseaux souterrains. Le phénomène, denommé par lui «la corrosion régressive», part donc des exurgences vers des ponors, mais il peut également naître d'une galerie plus large, où se trouve une cascade, qui peut créer une dépression.

Bien qu'intéressant comme idée, le mécanisme imaginé par Knebel est impossible dans la forme conçue par lui, car conformément à l'équation de Bernoulli, la dépression ne peut guère actionner sur plus de $10 \mathrm{~m}$, cas-limite correspondant à une colonne d'eau exerçant une pression égale à la pression atmosphérique. Le développement théoretique du problème a été fait à une autre occasion (Bleahu, 1957); nous n'y revenons plus ici.

Mais, quelle que soit la distance sur laquelle peut actionner l'absorption dans l'hypothèse de Knebel, elle s'exerce sur un cours d'eau se trouvant dans un canal affluent; or, nous devons expliquer justement le mécanisme qui a crée ce canal et la confluence avec la rivière principale.

Le problème trouve une solution si l'on considère les fissures capillaires où l'eau pénétrant de l'extérieur est stagnante, étant également saturée. Si l'on suppose qu'un tel tube capillaire se termine dans un canal par lequel l'eau s'écoule sous pression, à un endroit où le canal se rétrecit, la diminution hydrostatique locale exercera une dépression qui entraînera la première goutte d'eau du capillaire. Par son absorption toute la colonne de liquide du capillaire est mise en mouvement et par l'orifice d'entrée pénètre en retour de l'eau fraîche. Celle-ci rencontrant pour la première fois le calcaire, le dissout jusqu'à la saturation, restant ensuite chimiquement inactive. Mais, par l'absorption continue exercée par le canal sous pression, la colonne d'eau du capillaire tout entier est continuellement en mouvement, ce qui permet à l'eau fraîche d'entrer par l'orifice extérieur, en dissolvant progressivement les parois du tube. Le processus part donc de l'intérieur vers l'extérieur, étant conditionné par l'absorption du canal principal; la corrosion n'est cependant guère régressive, car elle progresse de l'extérieur vers l'intérieur. 
Nous avons denommé ce type de capture (Bleahu, .1957) capture karsto-sacuumatique ou simplement capture vacuumatique, démontrant par ceci qu'à la base du processus réside un phénomène identique à celui de l'absorption des pompes aspirantes. Nous désignons donc les confluences résultant d'un tel processus par le terme ce confluences vacuumatiques. Il parait être le seul mécanisme menant à ]'organisation d'un réseau hydrographique souterrain, comme conséquence d'un phénomène lié à la circulation même de l'eau dans le kars.t. La capture vacuumatique detient aussi un rôle important dans la création de tous les confluences épigénétiques mentionnées antérieurement, étant l'unique voie par laquelle les fissures capillaires peuvent être utilisées dans le karst; elle peut seule expliquer les confluences systématiques des canaux souterrains.

\section{Conclusions}

Les faits établis nous conduisent à la classification suivante des confluences souterraines:

\section{Confluences dues à l'intersection des voies initiales}

1. Confluences déterminées par la fissuration de la roch€:

- confluences en réseau-labyrinthe

- confluences en réseau dendritique

2. Confluences déterminées par des dislocations disjonctives

- confluences en réseau orthogonal ou rectangulaire

3. Confluences déterminées par des éléments de stratification

- confluences sur des jointures de couches

- confluences de contact stratigraphique

II. Confluences dues à la position dans l'espace des voies initiales

1. Confluences par surcreusement

- confluences antécédentes

- confluences épigénétiques

2. Confluences par creusement latéral

- confluences antécédentes

- confluences épigénétiques

3. Confluences par haussement de l'espace vide

- confluences par érosion remontante

- confluences par effondrement 
III. Confluences dues aux processus hydrodynamiques

- confluences vacuumatiques

En présence des cas concrets il n'est évidemment pas toujours facile de préciser la genèse et le type auquel appartient une confluence souterraine; ceci pour plusieurs raisons, à savoir:

1. Le plus souvent il ne s'agit pas d'un facteur unique, mais d'un complexe de causes. Ainsi, par ex., à n'importe quelle confluence épigénétique peut s'associer un processus vacuumatique.

2. Il peut exister une convergence morphologique, ayant toutefois à la base des processus génétiques différents. Ainsi, par ex., dans les galeries actives débouchant dans un gouffre, la confluence peut être de nature tectonique, ou due à l'érosion remontante (B. Gèze, 1953, fig. $6 \mathrm{~B}$ ), qui peut découvrir des voies préexistantes ou réactiver des fissures capillaires, ou bien cette confluence peut provenir d'un stade initial de capillaires sous charge.

3. Le plus souvent, pour expliquer un réseau souterrain complexe, à confluences multiples, il faut remonter dans le temps jusqu'au stade de réseau sous charge ou bien même de fissures capillaires. Ce que nous remarquons est le résultat d'une évolution prolongée, qui a amené des modifications profondes, dénaturant les aspects initiaux. De même que dans toutes les géo-sciences, l'analyse ne doit jamais s'arrêter aux aspects actuels mais au contraire, elle doit remonter le temps afin de surprendre le mécanisme intime du phénomène.

La classification des confluences souterraines présentée n'est qu'un premier essai. Il existe peut-être aussi d'autres modalités de génèse; les faits étant établis et les hypothèses émises, on pourrait faire aussi un autre groupement, plus pratique. Mais le problème n'est pas là. Ce que nous avons voulu démontrer, c'est que les confluences souterraines représentent un problème compliqué, qui marque une fois de plus la différence fondamentale qui existe entre les processus morphogénétiques endokarstiques et ceux qui se produisent à la surface de la terre.

\section{RÉSUMÉ}

La constitution d'un réseau hydrographique souterrain dépend de la mesure dans laquelle peuvent se réaliser des confluences entre différents cours d'eau. Contrairement à la surface, où les confluences sont déterminées par des processus propres à l'écoulement-même de l'eau, dans le karst souterrain les confluences ont un caractère fortuit et sont redevables à certaines circonstances indépendantes du phénomène d'écoulement. Ces caractères sont: préexistence des voies de circulation et le mode de leur distribu- 
tion spatiale. Il faut y ajouter un processus propre à la circulation endokarstique déterminée par l'écoulement sous charge et qui est es seul capable d'expliquer les confluences systématiques souterraines. En fonction de ces facteurs on propose une classification morphogénétique des confluences souterraines.

\section{ZUSAMMENFASSUNG}

Die Entstehung der unterirdischen Wassernetze ist von der Möglichkeit, in der sich unterirdische Zusammenflüsse bilden können, abhängig. Im Gegensatz zu der Erdoberfläche, wo die Zusammenflüsse von eigenartigen Vorgängen des Wasserfließens bedingt sind, haben die unterirdischen Zusammenflüsse einen zufälligen Charakter. Sie sind von der Mitwirkung einiger von dem Wasserfließen unabhängigen Umstände kedingt. Diese Umstände sind: das Vorhandensein vorherbestehender Fliєßbahnen und ihre Raumverteilung. Dazu kommt noch ein vom Druckfließen bedingter und dem unterirdischen Fließen eigener Vorgang, der einzig imstande ist, die systematischen unterirdischen Zusammenflüsse zu erklär€n.

Unter Berücksichtigung dieser Faktoren wird eine morphologisch-genetische Klassifikation der endokarstischen Zusammenflüsse vorgeschlagen.

\section{B I B LIOGRAPH IE}

Bleahu, M. (1957) - La capture karstique et son importance pour l'évolution morphologique des régions karstiques (en roumain avec résumé français). Probleme de Geografie, Vol. V, Bucarest.

GÈze, B. (1953) - La genèse des gouffres. I. Congrès Intern. Spźl., T. I, Paris. Kne B EL, W. (1906) - Höhlenkunde. Die Wissenschaft, H. 15, Braunschweig.

Lenmann, O. (1932) - Die Hydrographie des Karstes. Enzyklopädie der Erdkunde, Leipzig.

Macar, P. (1946) - Principes de géomorphologie normale. Liège.

Renault, Ph. (1958) - Eléments de spéléomorphologie karstique. Ann. de Spél. T. XIII, Paris. 